\title{
Stability and Stabilization of Distributed time Delay Systems
}

\author{
Frédéric Gouaisbaut
}

\begin{abstract}
This paper is dedicated to the stability and stabilization of state-distributed delay systems. The key idea is to express the distributed delay system as a barycentric sum of linear pointwise time delay systems. By using this reformulation, new stability criterion is proposed and is formulated in the form of Linear Matrix Inequality. These conditions for the stability of the system are obtained by using a Lyapunov Krasovskii functional . Based on this stability criterion, new types of controllers, taking into account the delayed part, are designed to ensure the asymptotic stability of the system. Several examples illustrate the proposed method.
\end{abstract}

Index Terms-Distributed delay systems, Stabilization, LMIs.

\section{INTRODUCTION}

Functional differential equations of retarded type with distributed delay are often used for the modelisation of many practical issues : biology, economic, ecology (see [12] for many examples). Concerning the stability and stabilization of such systems, several approaches have been proposed. The first one generally involves some Lyapunov-Krasovskii functional adapted to distributed time delay systems [6], [7], [10], [13]. Nevertheless, these techniques provide only sufficient conditions and they entail a conservatism which comes from the choice of the Lyapunov functional and the way to bound cross terms which appear in the derivative of the Lyapunov functional. Another method, which uses a Lyapunov-Razumikhin function [13], [14] allows to study systems with time varying delays. Furthermore, in order to reduce the conservatism of these technics, Gu et al [5], [6], [7] have proposed a discretization scheme for the general form of the Lyapunov Krasovskii introduced by [9]. In return of an improvement of the results, the computational effort is increasing. Another way to reduce the conservatism has been proposed by Fridman et al [3], [4]. Based on a descriptor model transformation, a new Lyapunov-Krasovskii functional is introduced which significantly improve the stability estimate. Another idea is to use a comparison principle [2], [15], which consists of replacing the original system by another one, simpler to study. The stability criterion is expressed in terms of matrix measures and matrix norms. Even if the results are easy to check, many variables have to tuned in order to optimise the delay upperbound. As technics inherited from matrix measures, this method allow to study the case of coefficients which are varying with the distributed delay. Finally, a original idea has been proposed by [11], who has extended Popov theory to time delay systems. The resulting criterion is then formulated in a Linear Matrix inequalities.

Frédéric Gouaisbaut is with the LAAS-CNRS, 7 avenue du Colonel Roche, 31077 Toulouse cedex 4, France. fgouaisb@laas.fr
In this paper, we propose a new robust stability condition for distributed system, which can be solved very efficiently by semi-definite solvers (see [1]). This criterion takes into account the case of coefficients variable with the distributed delay. Based on this result, different types of controllers are derived, which stabilize the system for all delays less than a prescribed upper bound.

The paper is organized as follows : after some assumptions, the section III proposes to transform the system into a new system, easier to study. In section IV, based on this transformation, by using a Lyapunov-krasovskii approach, we provide a new delay dependent stability criterion. Some examples and comparisons allow to show the efficiency of the algorithm.

The section $\mathrm{V}$ is devoted to the stabilization of a distributed system. Two controllers are proposed : the first one takes account of the delay size, whereas the second one is a memoryless state feedback. Finally, the last section provide some examples.

Notations: Throughout the paper, the following notations are used. For a two symmetric matrices, $A$ and $B, A>(\geq) B$ means that $A-B$ is (semi-) positive definite. $A^{T}$ denotes the transpose of $A, I_{n}$ and $0_{m, n}$ denote the respectively the identity matrix of size $n$ and null matrix of size $n \times n$. If the context allows it the dimensions of these matrices are often omitted.

\section{Assumptions}

Consider the following time delay system

$$
\left\{\begin{array}{c}
\dot{x}(t)=(A+\triangle A) x(t)+\left(A_{r}+\triangle A_{r}\right) x(t-h) \\
+\int_{-\tau}^{0} A_{d}(w) x(t+w) d w+B u(t), t>0, \\
x(t)=\phi(t), \text { for } t \in[-\delta, 0], \delta=\max (h, \tau)
\end{array}\right.
$$

where $\tau, h$ are constant delays, $x(t) \in \mathbb{R}^{n}, A, A_{r} \in$ $\mathbb{R}^{n \times n}, B \in(R)^{n \times m}$ are known constant matrices, $A_{d}$ is a function from $[-\tau, 0]$ to $\mathbb{R}^{n} \cdot u \in \mathbb{R}^{m}$ is the control vector. We denote the state of the system $x_{t}$ as :

$$
x_{t}(.):\left\{\begin{aligned}
{[-h, 0] } & \rightarrow R^{n} \\
\theta & \mapsto x_{t}(\theta)=x(t+\theta)
\end{aligned}\right.
$$

The uncertainties $\triangle A$ and $\triangle A_{r}$ satisfy the following normbounded conditions :

$$
\begin{aligned}
\triangle A & =D F(t) E, \text { where } F^{T}(t) F(t) \leq I, \\
\triangle A_{r} & =D_{r} G(t) E_{r}, \text { where } G^{T}(t) G(t) \leq I,
\end{aligned}
$$

where $D, D_{r} \in \mathbb{R}^{n \times n}$. $E, E_{r} \in \mathbb{R}^{n \times n}$ are constant known weighting matrices. 
We assume that function :

$$
A_{d}\left\{\begin{array}{l}
{\left[-\tau_{\text {sup }}, 0\right] \underset{A^{\prime}}{\longrightarrow} \longrightarrow \mathbb{R}^{n \times n}} \\
w \longmapsto A_{d}(w)
\end{array}\right.
$$

satisfies the following property:

$$
\forall \omega \in\left[-\tau_{\text {sup }}, 0\right], \exists \gamma>0,\left\|A_{d}(\omega)\right\|_{\infty}<\gamma .
$$

\section{BARYCENTRIC FORMULATION OF THE ORIGINAL SYSTEM}

the aim of this section is to find a polytopic modelisation of system (1) in order to developp delay dependent stability criterion. We propose the following lemma.

Lemma 1: For a given $\tau_{\text {sup }}$, System (1) can be transformed into:

$$
\left\{\begin{array}{l}
\dot{x}(t)=(A+\Delta A) x(t)+\left(A_{r}+\Delta A_{r}\right) x(t-h) \\
+\int_{-\tau}^{0} \sum_{i=1}^{r} h_{i}(w) A_{d i} x(t+w) d w+B u(t)+f_{1}\left(t, x_{t}\right), \\
x(t)=\phi(t), \text { for } t \in[-\tau, 0] .
\end{array}\right.
$$

where the functions

$$
h_{i}\left\{\begin{array}{c}
{\left[-\tau_{\text {sup }}, 0\right] \longrightarrow[0,1]} \\
w \longmapsto h_{i}(w)
\end{array}, \forall i \in\{1, . ., r\},\right.
$$

are piecewise continuous and verify the convex sum property $\sum_{i=1}^{r} h_{i}(w)=1$.

Proof: For a given $h_{\text {sup }}$, function $A_{d}$ is a continuous function and using property (3), we know that $\forall(i, j) \in$ $1, \ldots, n,\left|A_{d i j}\right| \leq \gamma$ which implies that function $A_{d}$ is belonging to an hypercube $\mathcal{C}$. Actually, it is not the better one, since it always exists a minimal convex set $\mathcal{P}$ included in $\mathcal{C}$.

Consequently, we define the convex hull associated to function $A_{d}$ by the minimal convex set spanned by matrices $\left\{A_{d 1}, \ldots, A_{d r}\right\}$ such that we can find $r$ functions $h_{i}$, which satisfy a convex sum property $\sum_{i=1}^{r} h_{i}(w)=1, h_{i} \geq 0, \forall i \in$ $\{1, \ldots, r\}$. with $A_{d}(\theta)=\sum_{i=1}^{r} h_{i}(\theta) A_{d i}$ for $\theta \in\left[-\tau_{\text {sup }}, 0\right]$

Remark 2: By construction, the matrices $A_{d i}$ and functions $h_{i}$ generally depend explicitly on the value of $\tau_{\text {sup }}$. The degree of conservatism introduced by this modelisation will depend on the gap between the delay upper bound of the system $\tau_{\text {sup }}$, and the real delay $\tau$ of the process.

In the following section, we consider the autonomous system, and we provide a new stability criterion.

\section{STABILITY OF DISTRIBUTED DELAY SYSTEMS}

Let consider the following autonomous system :

$$
\left\{\begin{aligned}
\dot{x}(t)= & (A+\Delta A) x(t)+\left(A_{r}+\Delta A_{r}\right) x(t-h) \\
& +\int_{-\tau}^{0} A_{d}(w) x(t+w) d w, t>0, \\
& x(t)=\phi(t), \text { pour } t \in[-\delta, 0] .
\end{aligned}\right.
$$

According to Lemma 1, it can be transformed into the following form :

$$
\begin{aligned}
x(t)= & (A+\Delta A) x(t)+\left(A_{r}+\Delta A_{r}\right) x(t-h) \\
& +\int_{-\tau}^{0} \sum_{i=1}^{r} h_{i}(w) A_{d i} x(t+w) d w,
\end{aligned}
$$

where the functions $h_{i}, \forall i \in\{1 \ldots r\}$ verify the two properties $h_{i} \geq 0, \forall i \in\{1 \ldots r\}$ and $\sum_{i=1}^{r} h_{i}(w)=1$.

The following theorem gives a first result on the delay dependent stability for system (6).

Theorem 3: System (5) is asymptotically stable for all delays $\tau \in\left[0, \tau_{\text {max }}\right]$, if there exists $S>0, T>0, \bar{R}>0$ of appropriate dimensions and scalars $\alpha>0, \beta>0$ satisfying the following optimization problem :

$$
\tau_{\max }=\max _{S, \bar{R}, T, \alpha, \beta}(\tau),
$$

submitted to constraints:

$$
\Theta_{i}=\left[\begin{array}{cccccc}
\Gamma & A_{r} S & S E^{T} & S E_{r}^{T} & \tau A_{d i} & 0 \\
S A_{r}^{T} & -T & 0 & 0 & 0 & 0 \\
E S & 0 & -\alpha I_{n} & 0 & 0 & 0 \\
E_{r} S & 0 & 0 & -\beta I_{n} & 0 & 0 \\
\tau A_{d i}^{T} & 0 & 0 & 0 & -2 I_{n} & S \\
0 & 0 & 0 & 0 & S & -\bar{R}
\end{array}\right]<0
$$

$\forall i \in\{1, \ldots, r\}$

with $\Gamma=S A^{T}+A S+\alpha D D^{T}+\beta D_{r} D_{r}^{T}+T+\bar{R}$

Proof: Define the following Lyapunov-Krasovskii functional for system (6) :

$$
\begin{aligned}
V\left(x_{t}\right)= & x^{T} P x+\int_{t-\tau}^{t} \int_{s}^{t} x(w)^{T} Q x(w) d w d s \\
& +\int_{t-h}^{t} x^{T}(s) P T P x(s) d s \\
+ & \beta^{-1} \int_{t-h}^{t} x^{T}(s) E_{r}^{T} E_{r} x(s) d s,
\end{aligned}
$$

Remark that since $P, Q, T>0$ and $\beta>0$, we can conclude that for some $\epsilon>0$, the Lyapunov-Krasovskii functional condition $V\left(x_{t}\right) \geq \epsilon\left\|x_{t}(0)\right\|$ is satisfied (see [8]). The derivative of (8) along the solutions of system (6) leads to the following equality :

$$
\begin{aligned}
& \dot{V}\left(x_{t}\right)=x^{T}(t)\left(A^{T} P+P A+P T P+2 P \Delta A\right) x(t) \\
& +\tau x(t)^{T} Q x(t)+2 x^{T}(t) P \Delta A_{r} x(t-h) \\
& +2 x^{T}(t) P \int_{-\tau}^{0} \sum_{i=1}^{r} h_{i}(w) A_{d i} x(t+w) d w \\
& -\int_{t-\tau}^{t} x(w)^{T} Q x(w) d w \\
& -x_{t}^{T}(-h) P T P x_{t}(-h)+2 x^{T}(t) P A_{r} x_{t}(-h) \\
& +\beta^{-1}\left(x^{T}(t) E_{r}^{T} E_{r} x(t)-x_{t}^{T}(-h) E_{r}^{T} E_{r} x_{t}(-h)\right)
\end{aligned}
$$

Defining $S=P^{-1}$, and using the well known inequality $2 x^{T} y \leq x^{T} M x+y^{T} M^{-1} y, \forall M>0$, the cross terms can be bounded as follows: 


$$
\begin{aligned}
2 x^{T}(t) P A_{r} x_{t}(-h) \leq & x^{T}(t) P A_{r} S T^{-1} S A_{r}^{T} P x(t) \\
& +x_{t}^{T}(-h) P T P x_{t}(-h), \\
2 x^{T}(t) P \Delta A x(t) \leq & x^{T}(t)\left(\alpha P D D^{T} P\right. \\
& \left.+\alpha^{-1} E^{T} E\right) x(t) \\
2 x^{T}(t) P \Delta A_{r} x_{t}(-h) \leq & \beta x^{T}(t) P D_{r} D_{r}^{T} P x(t) \\
& +\beta^{-1} x_{t}(-h)^{T} E_{r}^{T} E_{r} x_{t}(-h)
\end{aligned}
$$

We get also the following inequality:

$$
\begin{gathered}
2 x^{T}(t) P \int_{-\tau}^{0} \sum_{i=1}^{r} h_{i}(w) A_{d i} x(t+w) d w \leq \\
\int_{-\tau}^{0} \sum_{i=1}^{r} h_{i}(w) x(t) P A_{d i} Q^{-1} A_{d i}^{T} P x(t) \\
+x(t+w)^{T} Q x(t+w) d w .
\end{gathered}
$$

Therefore, we obtain:

$$
\dot{V}\left(x_{t}\right) \leq x^{T} C(\tau) x(t)
$$

with

$$
C(\tau)=\left(\begin{array}{c}
A^{T} P+P A+\tau Q+P A_{r} S T^{-1} S A_{r}^{T} P \\
\sum_{i=1}^{r}\left(\int_{-\tau}^{0} h_{i}(w) d w\right) P A_{d i} Q^{-1} A_{d i}^{T} P \\
+P T P+\alpha P D D^{T} P \\
+\alpha^{-1} E^{T} E+\beta P D_{r} D_{r}^{T} P+\beta^{-1} E_{r}^{T} E_{r}
\end{array}\right) .
$$

System (6) is then asymptotically stable $\forall \tau \leq \tau_{\max }$ if $\forall \tau \leq$ $\tau_{\max }, C(\tau)$ is a negative definite matrix. Furthermore, it also proves that system (5) is asymptotically stable. Nevertheless, since it depends on the functions $h_{i}$, it is not easy task to test if $C(\tau)<0$. In order to construct a systematic criterion, let remark that $C(\tau)<0$ is equivalent to:

$$
S C(\tau) S<0, \text { avec } S=P^{-1} .
$$

Noting $R=S Q S$,

$$
S C(\tau) S<0
$$

is equivalent to

$$
\sum_{i=1}^{r} \chi_{i}\left(\tau^{-1}\left(\begin{array}{c}
S A^{T}+A S+T \\
+\tau R+A_{r} S T^{-1} S A_{r}^{T} \\
+\alpha D D^{T}+\alpha^{-1} S E^{T} E S \\
+\beta D_{r} D_{r}^{T}+\beta^{-1} S E_{r}^{T} E_{r} S
\end{array}\right)+\Delta_{i}\right)<0
$$

where $\Delta_{i}=A_{d i} S R^{-1} S A_{d i}^{T}$ and $\chi_{i}=\left(\int_{-\tau}^{0} h_{i}(w) d w\right)$.

So, if the inequalities

$$
\left(\tau_{\max }^{-1}\left(\begin{array}{c}
S A^{T}+A S+\tau_{\max } R \\
+A_{r} S T^{-1} S A_{r}^{T}+T \\
+\alpha D D^{T}+\alpha^{-1} S E^{T} E S \\
+\beta D_{r} D_{r}^{T}+\beta^{-1} S E_{r}^{T} E_{r} S
\end{array}\right)+\Delta_{i}\right)<0,
$$

are satisfied $\forall i \in\{1, \ldots, r\}$, then $S C\left(\tau_{\max }\right) S<0$ and system (6) is asymptotically stable for all delays $\tau \in\left[0, \tau_{\max }\right]$. Furthermore, it can be easily seen that if the asymptotic stability is proved for a delay $\tau$, the result holds also for any smaller delay.

Using notations already defined, inequalities (10) can be written as:

$$
\begin{gathered}
\Gamma+A_{r} S T^{-1} S A_{r}^{T} \\
+\alpha^{-1} S E^{T} E S+\beta^{-1} S E_{r}^{T} E_{r} S+\Delta_{i}<0
\end{gathered}
$$

First, by a simple change of variable,

$$
\bar{R}=\tau_{\max } R
$$

we have :

$$
\begin{gathered}
\Gamma+A_{r} S T^{-1} S A_{r}^{T} \\
+\alpha^{-1} S E^{T} E S+\beta^{-1} S E_{r}^{T} E_{r} S+\Delta_{i}<0
\end{gathered}
$$

and

$$
V=\left[\begin{array}{llllll}
I_{n} & A_{r} S T^{-1} & \frac{S E^{T}}{\alpha} & \frac{S E_{r}^{T}}{\beta} & \tau_{\max } A_{d i} & D_{i}
\end{array}\right]_{(13)}
$$

where $D_{i}=\tau_{\max } A_{d i} S(\bar{R})^{-1}$, we can easily show that (12) is equivalent to:

$$
V \Theta_{i} V^{T}<0, \forall i \in\{1 \ldots r\}
$$

So, if inequalities $\Theta_{i}<0, \forall i \in\{1 \ldots r\}$ are satisfied, then it implies that inequalities (12) also hold. It also implies that $C(\tau)<0$, and that $\dot{V}\left(x_{t}\right) \leq \epsilon\left\|x_{t}(0)\right\|$, for some $\epsilon>0$, which concludes the proof.

The following examples show the effectiveness of the proposed technic.

\section{Examples and comparisons :}

Example 4: Let us consider (5) with :

$$
\begin{aligned}
A & =\left(\begin{array}{ccc}
-2 & 0 & 1 \\
1.75 & -1 & 0.8 \\
-1 & 0 & -1
\end{array}\right), \\
A_{d}(w) & =\left(\begin{array}{ccc}
-1 & 0 & 0 \\
-0.1 & 0.25 \cos (w) & 0.2 \\
-0.2 & 1 & 1
\end{array}\right), \\
A_{r} & =\left(\begin{array}{ccc}
0.1 & 0 & 0 \\
0 & 0.1 & 0 \\
0 & 0 & 0.1
\end{array}\right) .
\end{aligned}
$$

First, function $A_{d}(\omega)$ can be included into a polytopic set: indeed, we have the following equality $A_{d}(\omega)=h_{1}(\omega) A_{d 1}+$ $h_{2}(\omega) A_{d 2}$ with $h_{1} \omega=\frac{1-\cos (\omega)}{2}, h_{2}(\omega)=\frac{1+\cos (\omega)}{2}$ and

$$
\begin{aligned}
& A_{d 1}=\left(\begin{array}{ccc}
-1 & 0 & 0 \\
-0.1 & -0.25 & 0.2 \\
-0.2 & 1 & 1
\end{array}\right), \\
& A_{d 2}=\left(\begin{array}{ccc}
-1 & 0 & 0 \\
-0.1 & 0.25 & 0.2 \\
-0.2 & 1 & 1
\end{array}\right) .
\end{aligned}
$$

which show that $A_{d}(\omega)$ is belonging to the convex hull spanned by $\left(A_{d 1}, A_{d 2}\right)$. Using theorem 3 , we prove that system (1) is asymptotically stable for all delays $\tau \in[0,0.47]$. Based on the comparison principle, previous works (see [15]) find an upper bound of 0.0968 .

Remark 5: In this example, the function cos is a bounded function and do not depend on the chosen value $\tau_{\text {sup }}$. As a 
matter of fact, the vertices of the convex hull $A_{d 1}, A_{d 2}$ are independent of the value $h_{\text {sup }}$.

Example 6: This example illustrates the dependence between the value $\tau_{\text {sup }}$ and $\tau_{\max }$, solution of the Linear Matrix Inequalities (7). Let choose the following system :

$$
\dot{x}=-x(t)+0.1 x(t-h)+\int_{-\tau}^{0} \omega x(t+\omega) d \omega,
$$

The barycentric modelisation of function $A_{d}(\omega)$ leads to the following equality $\omega=h_{1}\left(-\tau_{\text {sup }}\right)+h_{2}(\omega) .0$. with $h_{1}(\omega)=$ $\frac{-\omega}{\tau_{\text {sup }}}, h_{2}(\omega)=\frac{\tau_{\text {sup }}+\theta}{\tau_{\text {sup }}}$. It is easy to show that $h_{1}$ and $h_{2}$ satisfy $h_{1 / 2}>0$ and $h_{1}+h_{2}=1$. The vertices of the convex hull are then defined by $A_{d 1}=-\tau_{\text {sup }}$ and $A_{d 2}=0$ and depend on $\tau_{\text {sup }}$.

Different choices of $\tau_{\text {sup }}$ and the resulting $\tau_{\max }$ are summarized in table I.

TABLE I

UPPERBOUND $\tau_{\text {max }}$ FOR DIFFERENT CHOICE OF $\tau_{\text {sup }}$
\begin{tabular}{|l|l|}
\hline$\tau_{\text {sup }}$ & $\tau_{\max }$ \\
\hline 5 & 0.15 \\
\hline 2 & 0.44 \\
\hline 1 & 0.89 \\
\hline 0.95 & 0.94 \\
\hline 0.949 & 0.949 \\
\hline
\end{tabular}

The result of the optimisation scheme show that theorem 3 can lead to a conservative result, i.e. a loose bound $\tau_{\max }$, compared with the initial chosen bound $\tau_{\text {sup }}$. Furthermore, it is obvious that for $\tau_{\text {sup } 1}>\tau_{\text {sup } 2}, \tau_{\max 1} \leq \tau_{\max 2}$ since the size of generated polytop is increasing. So, to improve the result, we decrease the size of the convex hull, i.e. the value of $\tau_{\text {sup }}$, to find the best $\tau_{\max }$. For this example, we find $\tau_{\max }=0.945$.Using a method proposed by [15] and based on comparison principle, we find that the system is asymptotically stable for $\tau \in[0 ; 0.90]$. For this example, there is no difference between our results and the result from [15] based on the use of matrix measures. Indeed, this approach is very efficient for systems of small dimensions.

\section{Stabilization OF DistRibuted DELAY SYSTEMS}

In this section, we consider system (4). The aim of this section is to develop two types of controllers : the first one is based on the knowledge of the functions $h_{i}$ and the value of the delay. The second one is designed, when the value of the delay $\tau$ is unknown.

A. Case of a known delay

We propose to design a controller of the form :

$$
u(t)=K x(t)+\int_{-\tau}^{0} \sum_{i=1}^{r} h_{i}(w) K_{i} x(t+w) d w .
$$

The system (4) with (14) is governed by the following differential equations :

$$
\begin{gathered}
\dot{x}(t)=(A+\Delta A-B K) x(t)+\left(A_{r}+\Delta A_{r}\right) x(t-h) \\
+\int_{-\tau}^{0} \sum_{i=1}^{r} h_{i}(w)\left(A_{d i}-B K_{i}\right) x(t+w) d w+B u(t), t>0,
\end{gathered}
$$

We propose the following theorem :

Theorem 7: System (15) with the control law (14) is asymptotically stable for all delays $\tau \in\left[0, \tau_{\max }\right]$, if there exists $S, \bar{R}, T>0$ of appropriate dimensions, $W, W_{1}, \ldots, W_{r} \in$ $\mathbb{R}^{m \times n}$ and scalars $\alpha>0, \beta>0$ satisfying the optimization problem :

$$
\tau_{\max }=\max _{S, \bar{R}, T, W, W_{1}, \ldots, W_{r}, \alpha, \beta}(\tau)
$$

submitted to the constraints:

$$
\Xi=\left[\begin{array}{cccccc}
\Gamma & A_{r} S & S E^{T} & S E_{r}^{T} & \tau I_{n} & 0 \\
S A_{r}^{T} & -T & 0 & 0 & 0 & 0 \\
E S & 0 & -\alpha I_{n} & 0 & 0 & 0 \\
E_{r} S & 0 & 0 & -\beta I_{n} & 0 & 0 \\
\tau I_{n} & 0 & 0 & 0 & -2 I_{n} & \kappa_{i} \\
0 & 0 & 0 & 0 & \left(\kappa_{i}\right)^{T} & -\bar{R}
\end{array}\right]<0
$$

$\forall i \in\{1, \ldots, r\}$, with $\kappa_{i}=A_{d} S-B W_{i}, \Gamma=S A^{T}+A S-$ $B W-W^{T} B^{T}+\alpha D D^{T}+\beta D_{r} D_{r}^{T}+T+\bar{R}$.

The gains of the controller are then given by

$$
\begin{aligned}
K & =W S^{-1} \\
K_{i} & =W_{i} S^{-1}, \forall i \in\{1, \ldots, r\}
\end{aligned}
$$

Proof: By using theorem 3, system (15) is asymptotically stable if there exists $S, R, T \in \mathbb{R}^{n \times n} 3$ positive definite matrices, and $\alpha, \beta$ two positive reals such that $\Gamma=S A^{T}+A S-B W-W^{T} B^{T}+\alpha D D^{T}+\beta D_{r} D_{r}^{T}+$ $T+\bar{R}, \delta_{i}=\left(A_{d i} S-B W_{i}\right) \bar{R}^{-1}\left(A_{d i} S-B W_{i}\right)^{T}$.

Let $W=K S, W_{i}=K_{i} S, \bar{R}=\tau_{\max } R$ and

$$
Z=\left[\begin{array}{llllll}
I_{n} & A_{r} S T^{-1} & \frac{S E^{T}}{\alpha} & \frac{S E_{r}^{T}}{\beta} & \tau_{\max } I_{n} & D_{i}
\end{array}\right]
$$

where $D_{i}=\tau_{\max } A_{d i} S(\bar{R})^{-1}$

We show that inequation (12) is equivalent to:

$$
Z \Xi Z<0
$$

This last inequality conludes the proof

\section{B. Case of a unknown delay}

In this case, we propose to design a controller of the form:

$$
u(t)=K x(t)
$$

The system (4) with (18) is governed by the following differential equations :

$$
\begin{aligned}
& \dot{x}(t)=(A+\Delta A-B K) x(t)+\left(A_{r}+\Delta A_{r}\right) x(t-h) \\
& +\int_{-\tau}^{0} \sum_{i=1}^{r} h_{i}(w) A_{d i} x(t+w) d w+B u(t), t>0,
\end{aligned}
$$

We have the following corollary :

Corollary 8: System (19) with control law (18) is asymptotically stable for all delays $\tau \in\left[0, \tau_{\max }\right]$, if there exist $S, \bar{R}, T>0$ of appropriate dimensions, $W \in \mathbb{R}^{n \times n}$ and $\alpha, \beta>0$ satisfying the optimization problem :

$$
\tau_{\max }=\max _{S, \bar{R}, T, W, \alpha, \beta}(\tau)
$$


submitted to the constraints :

$$
\left[\begin{array}{cccccc}
\Gamma & A_{r} S & S E^{T} & S E_{r}^{T} & \tau A_{d i} & 0 \\
S A_{r}^{T} & -T & 0 & 0 & 0 & 0 \\
E S & 0 & -\alpha I_{n} & 0 & 0 & 0 \\
E_{r} S & 0 & 0 & -\beta I_{n} & 0 & 0 \\
\tau A_{d i}^{T} & 0 & 0 & 0 & -2 I_{n} & S \\
0 & 0 & 0 & 0 & S & -\bar{R}
\end{array}\right]<0
$$

$\forall i \in\{1, \ldots, r\}$, with $\Gamma=S A^{T}+A S-B W-W^{T} B^{T}+$ $\alpha D D^{T}+\beta D_{r} D_{r}^{T}+T+\tau_{\max } R$. The gain of the controllers are then given by

$$
K=W S^{-1} \text {. }
$$

Proof: The proof of this corollary is based on the proof of theorem 7

\section{EXAMPLES}

Example 9: Let consider system (1) which has been studied by [11] :

$$
\begin{gathered}
\dot{x}(t)=(1+0.2 \sin (t)) x(t)+(1+0.2 \cos (t)) x(t-h) \\
+\int_{0}^{\tau} \theta x(t-\theta) d \theta+u(t)
\end{gathered}
$$

Here $D=D_{r}=0.2$ and $E=E_{r}=1$. The problem is to achieve the closed loop stability with a memoryless controller $u(t)=K x(t)$. Solving optimisation problem from theorem (7), we propose the following results in table II:

TABLE II

MAXIMUM DELAY FOR DIFFERENT CONTROLLERS

\begin{tabular}{|l|l|}
\hline$\tau_{\max }$ & $K$ \\
\hline 1.6 & -4.98 \\
\hline 2.7 & -9.55 \\
\hline 4.1 & -19.92 \\
\hline 6.1 & -39.9 \\
\hline 9.8 & -99.95 \\
\hline
\end{tabular}

Based on generalized Popov theory, the method of Ivanescu et al [11] yields to a controller $u(t)=-94.041 x(t)$ for a maximal delay of $4.21 \mathrm{~s}$.

Example 10: Let us consider system (1) with

$$
\begin{aligned}
A & =\left(\begin{array}{ccc}
-2 & 0 & 1 \\
1.75 & 0.25 & 0.8 \\
-1 & 0 & 1
\end{array}\right), \\
A_{d}(w) & =\left(\begin{array}{ccc}
-1 & 0 & 0 \\
-0.1 & 0.25 \cos (w) & 0.2 \\
-0.2 & 1 & 1
\end{array}\right) \\
A r & =\left(\begin{array}{ccc}
0.1 & 0 & 0 \\
0 & 0.1 & 0 \\
0 & 0 & 0.1
\end{array}\right), B=\left(\begin{array}{l}
0 \\
0 \\
1
\end{array}\right) .
\end{aligned}
$$

The weighting functions $h_{i}$ are the following $h_{1}(w)=$ $\frac{1-\cos (w)}{2}$, and $h_{2}(w)=\frac{1+\cos (w)}{2}$. The different vertices for the convex hull are the following :

$$
\begin{aligned}
A_{d 1} & =\left(\begin{array}{ccc}
-1 & 0 & 0 \\
-0.1 & -0.25 & 0.2 \\
-0.2 & 1 & 1
\end{array}\right) \\
A_{d 2} & =\left(\begin{array}{ccc}
-1 & 0 & 0 \\
-0.1 & 0.25 & 0.2 \\
-0.2 & 1 & 1
\end{array}\right)
\end{aligned}
$$

\section{Case of a known delay :}

Using theorem 7, we prove that the system (23) with (14) is asymptotically stable for $\tau \in[0,3.33]$.

\section{Case of an unknown delay :}

Using theorem 8, we prove that the system (23) with (18) is asymptotically stable for $\tau \in[0,2.29]$. Indeed, the use of a control law,taking into account the size of the delay allows to find a better upperbound for the delay.

\section{CONCLUSION}

This paper has provided a new technique for checking the stability of distributed state delay system. The main idea is to write the kernel of the distributed delay as a barycentric sum of constant matrices. By this way, a Lyapunov-Krasovskii functional is provided and lead to a delay dependent criterion expressed in terms of LMIs. Based on this result, two different controllers have been derived. The first controller, which takes account of the distributed part of the system can be used when the delay value is exactly known, while the second is a simple feedback controller. The effectiveness of our approach has been tested on several examples. Remark that this method is straightforward and can be extended to the case of time-varying delay.

\section{REFERENCES}

[1] S. Boyd, G. L. El, E. Feron, and V. Balakrishan. Linear Matrix Inequalities in System and Control Theory. SIAM, Philadelphia, 1994.

[2] M. Dambrine and J. Richard. Stability analysis of time-delay systems. Dynamic Syst. \& Applications, (2):405-414, 1993.

[3] E. Fridman. Stability of linear descriptor systems with delay: A lyapunov-based approach. Journal of Mathematical Analysis and Applications, 273(1):24-44, 2002.

[4] E. Fridman and U. Shaked. An improved stabilization method for linear time-delay systems. IEEE Trans. on Automatic control, 47(11):1931-1937, 2002.

[5] K. Gu. Discretized lmi set in the stability problem of linear uncertain time-delay systems. International Journal of Control, 68(4):923-934, 1997

[6] K. Gu. Discretization schemes for lyapunov-krasovskii functionals in time-delay systems. Kybernetika, 37(4):479-504, 2001.

[7] K. Gu, Q.-L. Han, A. C. J. Luo, and S.-I. Niculescu. Discretized lyapunov functional for systems with distributed delay and piecewise constant coefficients. International Journal of Control, 74(7):737-744, 2001

[8] K. Gu, K. Kharitonov, and J. Chen. Stability of Time-Delay Systems. Control Engineering Series. Birkhauser, Boston USA, 2003.

[9] E. Infante and W. Castelan. A lyapunov functional for a matrix difference-differential equation. Journal of Differential Equations, (29):439-451, 1978.

[10] D. Ivanescu. Sur la Stabilisation Des Systèmes À Retard : Théorie et Applications. PhD thesis, Institut National Polytechnique de Grenoble, 2000.

[11] D. Ivanescu, S.-I. Niculescu, J. Dion, and L. Dugard. Control of distributed delay systems with uncertainties : A generalised popov theory approach. Kybernetika, 37:325-343, 2001. 
[12] V. Kolmanovskii and A. Myshkis. Introduction to the theory and applications of functional differential equations. Kluwer Acad., Dordrecht, 1999.

[13] V. Kolmanovskii and J. Richard. Stability of some linear systems with delay. IEEE Trans. Aut. Control, 44(5):984-989, May 1999.

[14] S. Niculescu, C. De Souza, L. Dugard, and J. Dion. Robust exponential stability of uncertain systems with time-varying delays. IEEE Trans. Aut. Control, 43(5):743-748, May 1998.

[15] P. Tchangani. Sur la stabilité des systèmes héréditaires non linéaires. PhD thesis, LAIL, EC Lille, Univ. de Lille, France, Janvier 1999. 